\title{
A realidade da atenção a idosos portadores da doença de Alzheimer: uma análise a partir de idosos atendidos em serviços públicos
}

\author{
The reality of the elderly with Alzheimer's disease \\ caretaking: an analysis based on the elderly attended by \\ public services
}

\author{
Lilian de Oliveira Taylor ${ }^{1 *}$; Mara Solange Gomes Dellaroza ${ }^{2}$
}

Resumo

Com a elevação da expectativa de vida, temos um aumento na prevalência de doenças crônicodegenerativas, entre elas a doença de Alzheimer (DA). A DA é uma doença neurodegenerativa progressiva, responsável pela perda da função cognitiva e autonomia. Atualmente, não dispomos de medidas capazes de interromper ou modificar seu curso, então se torna indispensável a assistência interdisciplinar nos diferentes estágios. Os objetivos do estudo foram: identificar as características sócioeconômicas dos portadores de Alzheimer; descrever o processo de diagnóstico da doença; identificar os recursos terapêuticos utilizados pelos idosos. Estudo quantitativo, com entrevista a 22 idosos de um ambulatório público especializado, com aplicação da escala de Katz, Lawton, Mini Exame do Estado Mental (MEEM). Os idosos eram, na maioria, mulheres, casadas (50\%), com média etária de 80,2 anos. Os cuidadores eram mulheres (59\%), com média de idade de 61,5 anos, e a maioria eram filhos, seguidos pelos cônjuges. Dedicavam mais de oito horas diárias de cuidado ao idoso, sem rodízio com outros familiares e $50 \%$ dos idosos contavam com a ajuda financeira de filhos e apoio de grupos sociais. Sessenta e três $(63,6 \%)$ dos idosos apresentam dependência parcial para Atividade de Vida Diária Instrumental (AVDI). A maioria dos diagnósticos foi confirmada no ambulatório de especialidades por neurologistas. Os idosos usavam tratamento medicamentoso e nenhum realizava terapêuticas nãofarmacológicos.

Palavras-chave: Alzheimer. Processo de diagnóstico. Terapêuticas. Entidades de apoio social e financeiro.

\begin{abstract}
With the increase in life expectancy, there was an increase in the prevalence of chronic-degenerative diseases, including Alzheimer's disease (AD). The AD is a progressive neurodegenerative disease, responsible for the loss of cognitive function and autonomy. Currently, we have no measures to interrupt or modify its course; however, they are essential for an interdisciplinary assistance in different stages of the disease. The objectives of the study were: to identify the social and economic characteristics of individuals with Alzheimer's; describe the disease's diagnosis process; and identify the therapeutic resources used by the elderly. Interviews were carried out with 22 elderly in a specialized public clinic, using the scale of Katz, Lawton, Mini Mental State Exam (MMSE). Participants were mostly women, married (50\%), with an average age of 80.2 years. The caregivers were women (59\%), average age of 61.5 years, and mostly daughters, followed by spouse. They dedicated more than eight hours of daily care to the elderly, without rotation with other relatives, and $50 \%$ of the elderly relied on the financial help from their children and support from social groups. Sixty-three (63.6\%) of the elderly showed partial dependence on Intrumental Daily Life Activity (AVDI). Most diagnoses were confirmed at the clinic of specialties by neurologists. The elderly used drug treatment and no one use non-pharmacological treatment.
\end{abstract}

Key-words: Alzheimer's. Process diagnostic. Therapeutic. Social and financial support entities.

\footnotetext{
${ }^{1}$ Enfermeira pela Universidade Estadual de Londrina (UEL), Pós-graduação em Enfermagem Clínica Cirúrgica pela Universidade Federal de São Paulo (UNIFESP). E-mail: Lilian02_taylor@yahoo.com.br

* Autor (a) para correspondência

${ }^{2}$ Docente Assistente do Departamento de Enfermagem da Universidade Estadual de Londrina (UEL).
} 


\section{Introdução}

Com a elevação da expectativa de vida, verificada hoje em todo o planeta, um maior número de indivíduos alcança uma idade avançada e, como conseqüência, tem-se um aumento na prevalência de doenças crônico-degenerativas causadoras de demência (FRIDMAN et al., 2004).

O envelhecimento é um processo seqüencial, individual, acumulativo, irreversível, universal, não patológico, de deterioração de um organismo maduro, próprio a todos os membros de uma espécie, de maneira que o tempo o torne menos capaz de fazer frente ao estresse do meio-ambiente e, portanto, aumente sua possibilidade de morte (OPAS, 2003 apud BRASIL, 2006, p. 8).

No Brasil a lei $\mathrm{n}^{\circ} 8.842$, considera idoso pessoa maior de sessenta anos de idade (BRASIL, 1994).

Atualmente, estima-se que existam no Brasil cerca de 17,6 milhões de idosos. A previsão para o ano de 2050 é de que haverá cerca de dois bilhões de pessoas com sessenta anos e mais no mundo (BRASIL, 2006).

A Doença de Alzheimer é a causa mais comum de demência em idosos, e atinge cerca de $1 \%$ a $6 \%$ da população a partir dos 65 anos e 50\% em indivíduos com 95 anos ou mais (VILELA; CARAMELLI, 2006). No Brasil, os dados epidemiológicos são semelhantes. Em uma pesquisa realizada na cidade de Catanduva, estado de São Paulo, analisaramse 1660 pessoas com idade igual ou superior a 65 anos. Foram encontrados 118 casos de demência $(7,1 \%)$ sendo a Doença de Alzheimer responsável por 64 casos $(54,1 \%)$ (HERRERA JUNIOR; CARAMELLI; NITRINI, 1998).

O envelhecimento populacional não desencadeou apenas a mudança no perfil morbi-mortalidade, mas também trouxe consigo problemas sociais e financeiros de grandes proporções, relacionados à manutenção da saúde da população idosa. Nesse contexto, a Doença de Alzheimer figura como importante problema de saúde pública em todo mundo (MACHADO, 2006). Kwentus (2002 apud LUZARDO; WALDMAN, 2004, p. 136) cita que a "doença afeta 4 milhões de norte americanos e a previsão é de que afetará 8 milhões de pessoas no ano de 2040". Com base nesses fatos, é essencial que os profissionais de saúde conheçam a etiologia da Doença de Alzheimer, os sinais e sintomas, as formas de diagnóstico e terapêuticas indicadas em cada fase da doença.

A análise da doença de Alzheimer na comunidade é de extrema valia, devido a sua alta prevalência entre os idosos e ao fato de constituir importante causa de incapacidades nessa faixa etária. Há grandes lacunas estatísticas a respeito da situação da doença de Alzheimer no Brasil, e é importante uma investigação profunda dessa enfermidade, que já figura como um problema de saúde pública.

A doença de Alzheimer é a principal doença degenerativa cortical, ou seja, é caracterizada por perda de neurônios da substância cinzenta (GIROLAMI; ANTHONY; FROSCH, 2000), e acomete inicialmente a formação hipocampal, com posterior comprometimento de áreas corticais associativas e relativa preservação dos córtices primários (CARAMELLI; BARBOSA, 2002).

A Demência é caracterizada pela ocorrência de múltiplos déficits cognitivos que sejam suficientemente graves para interferir nas atividades profissionais, ocupacionais e sociais do indivíduo (BRASIL, 2006).

Verificou-se, nas últimas décadas, um grande avanço científico e tecnológico sobre a fisiopatologia da doença de Alzheimer e a descoberta de novos tratamentos, ainda não dispomos de medidas capazes de interromper ou modificar o curso da doença. Por esse motivo, diversas intervenções são essenciais para melhorar a qualidade de vida e a auto-estima de pacientes e familiares, e melhorar o desempenho cognitivo e funcional desses portadores. Por essa razão, é indispensável a assistência interdisciplinar 
nos diferentes estágios da doença. O paciente necessita de acompanhamento nutricional, psicológico, fisioterápico, além do atendimento da fonoaudióloga, serviço social, terapeuta ocupacional e da enfermagem (BOTTINO et al., 2002; WANNMACHER, 2005).

Além dos custos onerosos despendidos com a doença, o Alzheimer provoca danos emocionais, físicos, sociais e financeiros para os pacientes, familiares e cuidadores (MACHADO, 2006).

Cuidar de pacientes portadores da Doença de Alzheimer é uma das tarefas mais difíceis para a família, pois eles sofrem desgaste físico e emocional, o que leva a uma má qualidade de vida. Sendo assim, o atendimento à família cuidadora é crucial para o sucesso do tratamento. Os profissionais de saúde têm a função de orientar os cuidadores quanto à doença já que são responsáveis pelo cuidado do doente no domicílio. O doente precisa ser apoiado para que consiga viver a situação de forma mais tranqüila, a fim de garantir a dignidade do paciente (LUZARDO; WALDMAN, 2004).

O presente estudo teve como objetivos: identificar as características dos idosos acometidos pela moléstia, analisar o processo de diagnóstico e detecção precoce da doença, identificar os recursos terapêuticos e entidades de apoio utilizadas pelas famílias dos portadores de Alzheimer. Esperamos, assim, contribuir com a melhoria do atendimento aos pacientes com demência e estimular a implementação de uma rede de cuidados a portadores e familiares possibilitando uma maior qualidade de vida.

\section{Material e método}

O estudo foi desenvolvido com os pacientes de um ambulatório público especializado. A seleção dos indivíduos realizou-se conforme os seguintes critérios: 1) idade maior ou igual a 60 anos; 2) idoso com diagnóstico confirmado de Doença de Alzheimer a pelo menos um ano; 3) idoso não institucionalizado e residente na área urbana de
Londrina; 4) cuidador que tenha consentido em participar após explicação do objetivo e estratégias de coleta e tiver capacidade física e mental para responder as questões. Conforme consulta ao Setor de informática da Secretária Municipal de Saúde, estão inscritos no ambulatório acima citado 451 idosos, desde o ano de 2003. Foi realizado um sorteio aleatório de 106 idosos, o qual correspondeu a 23,5 $\%$ dos idosos inscritos, número que consideramos significativo para a proposta do estudo. Entretanto, devido a dificuldades operacionais, foram entrevistados somente 22 idosos, total que constitui uma população de conveniência.

O presente projeto foi aprovado pelo Comitê de pesquisa da UEL, parecer $n^{\circ} 184 / 07$. A Secretaria Municipal de Saúde de Londrina autorizou a realização do estudo.

Os cuidadores de idosos com Alzheimer foram entrevistados no domicílio. As entrevistas foram realizadas entre agosto e novembro de 2007 pela pesquisadora principal.

Seguindo um roteiro previamente elaborado com as seguintes partes: na primeira parte, referente à caracterização das condições de saúdo do idoso, realizou-se uma avaliação do nível de dependência do idoso através da escala de Katz e Lawton, respectivamente para atividades de vida diária e atividades instrumentais de vida diária, avaliação pelo Mini Exame do Estado Mental (MEEM) e comorbidades.

A segunda parte incluiu a caracterização do cuidador principal: quanto ao sexo, idade, grau de parentesco, tempo de estudo, questões sobre o processo de diagnóstico da doença (tempo, serviço) e uma avaliação das condições atuais de tratamento: terapias em realização (farmacológica e não farmacológica) e meio de acesso a estes tratamento.

Os dados coletados foram previamente categorizados, digitados em banco de dados no programa Excell 2003 e realizadas análises estatística descritivas. 
Tabela 1 - Caracterização socioeconômica dos idosos com Alzheimer. Londrina - 2007

\begin{tabular}{|c|c|c|}
\hline Variáveis & $n$ & $\%$ \\
\hline \multicolumn{3}{|l|}{ Sexo } \\
\hline Masculino & 9 & 40,9 \\
\hline Feminino & 13 & 59,1 \\
\hline \multicolumn{3}{|l|}{ Idade } \\
\hline 60-74anos & 3 & 13,6 \\
\hline $75-84$ & 14 & 63,6 \\
\hline$>85$ & 5 & 22,7 \\
\hline \multicolumn{3}{|l|}{ Estado Civil } \\
\hline Casado & 10 & 45,4 \\
\hline Separado & 1 & 4,5 \\
\hline Viúvo & 11 & 50,0 \\
\hline \multicolumn{3}{|l|}{ Situação Profissional } \\
\hline Pensionista & 2 & 9,1 \\
\hline Aposentado & 20 & 90,9 \\
\hline
\end{tabular}

Conforme a Tabela 1, a maior parte dos idosos, $13(59,1 \%)$, pertencia ao sexo feminino, média de idade de 80,2 anos, com $14(63,6 \%)$ de idosos na faixa compreendida entre 75 e 85 anos, viúvos $(50 \%)$ e aposentados (90,9\%). Em pesquisa semelhante realizada por Luzardo, Gorini e Silva (2006), observou-se que a maioria dos idosos era: mulher, casada e com média de idade de 75,19 anos, variando entre 61 e 86 anos. Os idosos também foram analisados em relação ao Mini-Exame do Estado Mental. O diagnóstico de demência, segundo a (DSM-IV), é fundamentalmente clínico, baseado na investigação cognitiva e funcional. Diversos instrumentos foram desenvolvidos para o rastreamento da demência em indivíduos de risco, como é o caso dos idosos. Entre estes testes, o Mini-Exame do Estado Mental (MEEM) é a escala mais amplamente empregada. O MEEM é um teste simples e rápido, que avalia orientação temporoespacial, memória, cálculo, linguagem e habilidades construtivas. As pontuações variam de 0 a 30 pontos. (FRIDMAN et al., 2004).
Almeida (1998) indica pontos de coortes diferenciados para o MEEM conforme escolaridade, e propõe o ponto de coorte 19/20 para idosos sem escolaridade e $23 / 24$ para idosos com histórico de escolaridade prévio.

Os resultados mostram um nível de escolaridade baixo, sendo 2 idosos analfabetos, 14 com menos de oito anos de estudo e 6 com mais de 8 anos.

A média do escore apresentado pelos pacientes foi de 15,4 , e $81,8 \%$ (18) dos idosos tiveram escores indicativos de déficit cognitivo, o que era esperado nesta situação. Os pacientes que apresentaram um ruim desempenho no MEEM devem ser submetidos à avaliação neuropsicológica por profissional especializado, com exames laboratoriais e de liquor, neuroimagem estrutural (tomografia computadorizada ou ressonância magnética) e funcional (tomografia de emissão de fóton único), eletro encefalograma, entre outros (NITRINI et al., 2005). 
Tabela 2 - Caracterização dos principais cuidadores e condições de cuidado, dos idosos com Alzheimer - Londrina, 2007.

\begin{tabular}{|c|c|c|}
\hline Variáveis & $n$ & $\%$ \\
\hline \multicolumn{3}{|l|}{ Sexo } \\
\hline Masculino & 9 & 40,9 \\
\hline Feminino & 13 & 59,1 \\
\hline \multicolumn{3}{|l|}{$\begin{array}{l}\text { Escolaridade } \\
\text { (anos de estudo) }\end{array}$} \\
\hline $0-7$ & 16 & 72,7 \\
\hline $8-12$ & 3 & 13,6 \\
\hline$>12$ & 3 & 13,6 \\
\hline \multicolumn{3}{|l|}{ Parentesco } \\
\hline Cônjuge & 9 & 40,9 \\
\hline Outros & 1 & 4,5 \\
\hline Filho & 12 & 54,5 \\
\hline \multicolumn{3}{|l|}{ Horas do cuidar } \\
\hline Até 4 horas & 1 & 4,5 \\
\hline Mais de 8 horas & 21 & 95,4 \\
\hline \multicolumn{3}{|l|}{ Rodízio } \\
\hline Sim & 9 & 40,9 \\
\hline Não & 13 & 59,1 \\
\hline \multicolumn{3}{|l|}{ Apoio financeiro } \\
\hline Sim & 11 & 50 \\
\hline Não & 11 & 50 \\
\hline \multicolumn{3}{|l|}{ Grupo de apoio } \\
\hline Sim & 12 & 54,5 \\
\hline Não & 10 & 45,4 \\
\hline
\end{tabular}

A Tabela 2 faz referência à caracterização do cuidador familiar. Aidade dos cuidadores variou entre o mínimo de 33 e o máximo de 83 anos, com média de 61,5 anos. Em relação à distribuição por gênero, 13 cuidadores eram mulheres enove homens. O nível de escolaridade foi baixo, com 16 cuidadores tendo de 0 a 7 anos de estudo, três cuidadores com oito a 12 anos de estudo e somente três com mais de 12 anos de estudo. Quanto à relação familiar dos cuidadores com os pacientes, $12(54,5 \%)$ dos cuidadores eram filhos e nove $(40,9 \%)$ cônjuges. Em relação ao cuidado, 21 cuidadores $(95,4 \%)$, dedicavam mais de oito horas de cuidado aos idosos, sendo que 13 cuidadores $(59,1 \%)$ relataram não ter rodízio para cuidar do doente. Metade dos idosos recebia algum tipo de ajuda financeira, principalmente dos filhos e 12 cuidadores $(54,5 \%)$ recebiam ajuda de grupos de apoio, especialmente de instituições religiosas da Associação Brasileira de Alzheimer.

Normalmente são as mulheres que assumem os cuidados, pois culturalmente é função desta cuidar dos filhos e do marido, além disso, presenciamos cuidadores idosos cuidando de idosos (SANTOS; PELZER; RODRIGUES, 2007). Segundo pesquisas, os cuidadores são em grande parte mulheres, representados por cônjuges ou filhas, com média de idade de 58,3 anos, e dedicam tempo integral ao cuidado com o idoso e têm escolaridade em torno de oito anos de estudo (VILELA; CARAMELLI, 2006). 
A baixa escolaridade afeta a qualidade da assistência prestada, pois o cuidador tem dificuldades em seguir dietas, ler prescrições e administrar medicamentos específicos em horários pré-determinados (NAKATANI et al., 2003).

Segundo Santos, Pelzer e Rodrigues (2007), a maior parte dos cuidadores tem religião definida, representando um mecanismo de enfrentamento das dificuldades, proporcionando bem-estar e tranqüilidade. Estes mesmos autores encontraram que seis cuidadores recebem ajuda para cuidar do idoso, três recebem auxílio de pessoas contratadas e familiares, três somente pessoas contratadas e dois são cuidadores exclusivos. Os familiares mais citados como ajudantes no cuidado foram os cônjuges, seguidos de filhas e irmãos.

Em outro estudo, que avaliou a sobrecarga dos cuidadores, demonstrou que a maior parte dos cuidadores, 55,6\%, apresentava sobrecarga moderada e $25,0 \%$ relataram sobrecarga moderada a severa (LUZARDO; GORINI; SILVA, 2006).

Esses dados revelam um grande impacto para a saúde do doente e do cuidador, pois estes, muitas vezes idosos, possuem grande sobrecarga de trabalho, e são os responsáveis pelos efeitos negativos da qualidade da assistência prestada ao idoso e do bem estar do cuidador. O excesso de atividades ocasiona nos cuidadores estresse físico, psicológico, financeiro e social, impossibilitando-os de se dedicarem a outros compromissos e realizarem atividades de lazer. Essas tensões provocam o desgaste e contribuem para o aparecimento de problemas de saúde no cuidador, e diversas vezes é necessária a institucionalização do doente por não ter mais condições de cuidá-lo (SANTOS, PELZER; RODRIGUES, 2007).

Dentre as dificuldades encontradas pelo familiar cuidador no processo de cuidar, temos a dificuldade no manejo com o doente, desconhecimento da doença, falta de divisão de tarefas com outros familiares, carência de serviços de saúde que ofereçam apoio e dificuldade financeira. Diante disso, é extremamente importante haver um rodízio entre os familiares para cuidar do idoso e não sobrecarregar apenas um membro da família (LUZARDO; WALDMAN, 2004; SANTOS; PELZER; RODRIGUES, 2007)

Atualmente, os serviços de saúde recomendam tratar do idoso no domicílio e repassam à família a responsabilidade de cuidar deste, a fim de diminuir onerosos gastos públicos com hospitalizações. Sendo assim, a família e os amigos desempenham papel indispensável no cuidado e reabilitação do idoso.

Tabela 3 - Atividades desenvolvidas pelos cuidadores dos idosos com Alzheimer. Londrina - 2007

\begin{tabular}{|c|c|c|}
\hline Variáveis & $\mathbf{n}$ & $\%$ \\
\hline \multicolumn{3}{|l|}{ Higiene } \\
\hline Sim & 6 & 27,3 \\
\hline Não & 16 & 72,7 \\
\hline \multicolumn{3}{|l|}{ Alimentação } \\
\hline Sim & 4 & 18,2 \\
\hline Não & 18 & 81,8 \\
\hline \multicolumn{3}{|l|}{ Movimentação } \\
\hline Sim & 7 & 31,8 \\
\hline Não & 15 & 68,2 \\
\hline \multicolumn{3}{|l|}{ Medicamento } \\
\hline Sim & 18 & 81,8 \\
\hline Não & 4 & 18,2 \\
\hline
\end{tabular}




\begin{tabular}{lcc}
\hline Curativo & & \\
Sim & 2 & 9,1 \\
Não & 20 & 90,9 \\
\hline Outras atividades & & \\
Sim & 8 & 36,4 \\
Não & 14 & 63,6 \\
\hline
\end{tabular}

Estudos realizados por Santos, demonstram alimentar-se e 14,3\% necessitam de auxílio para $42,8 \%$ dos idosos entrevistados necessitam de tarefas domésticas, como cozinhar, lavar e fazer auxílio em todas as tarefas, $21,4 \%$ precisam de ajuda para tomar banho, vestir e tirar a roupa e compras.

Tabela 4 - Distribuição dos idosos portadores de Alzheimer, segundo grau de dependência para as ABVD (Atividades básicas de vida diária). Londrina - 2007.

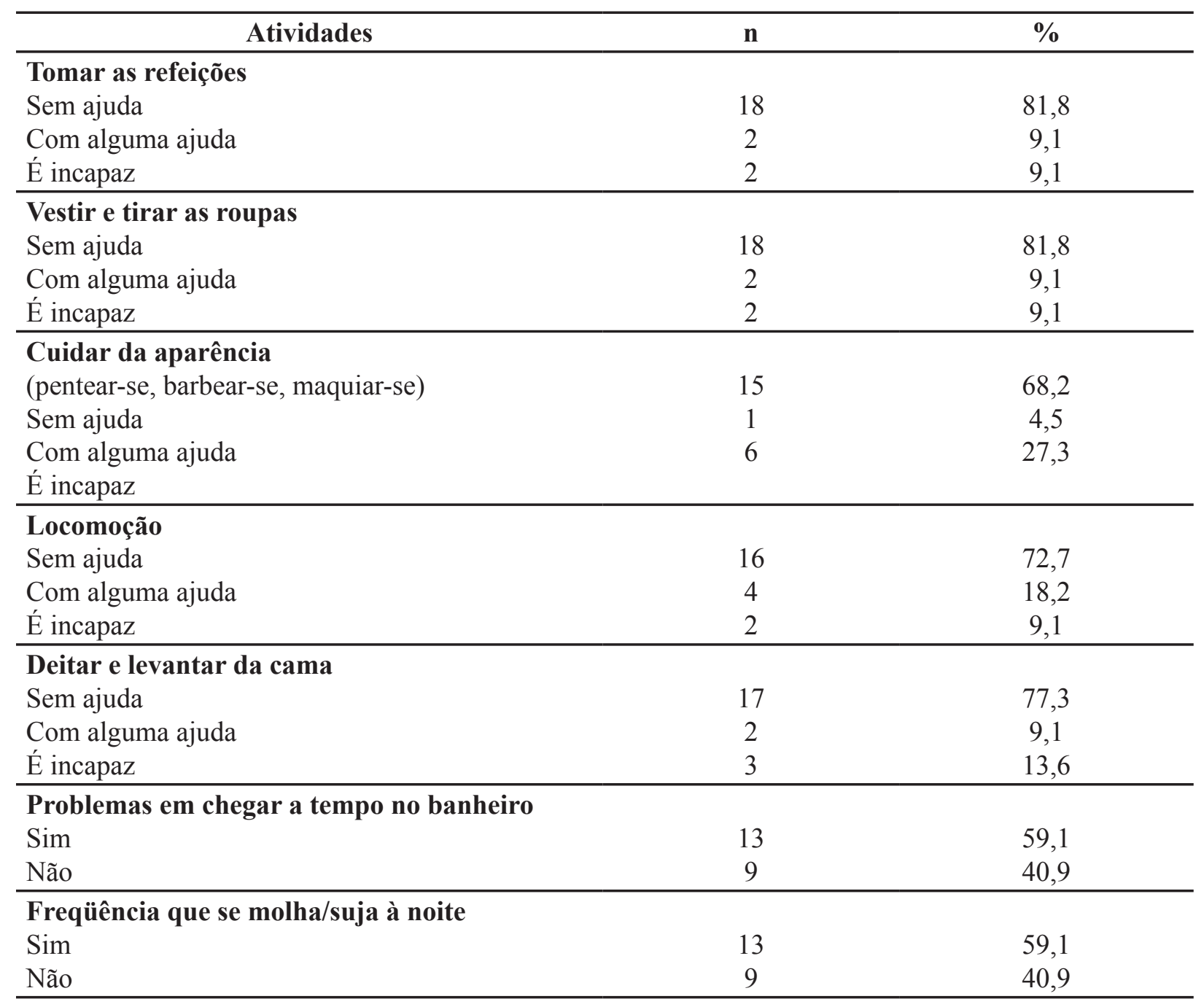


A Tabela 4 demonstra o grau de dependência dos pacientes eram totalmente dependentes para para as ABVD (Atividades básicas de vida as atividades básicas de vida diária (vestir-se, diária). Segundo Vilela e Caramelli (2006), 45\% alimentar-se, higiene pessoal).

Tabela 5 - Distribuição dos idosos portadores de Alzheimer, segundo grau de dependência para as AIVD (Atividades instrumentais da vida diária). Londrina - 2007.

\begin{tabular}{lcc}
\hline \multicolumn{1}{c}{ Atividades } & $\mathbf{n}$ & \% \\
\hline Usa o telefone & 7 & 31,8 \\
Sem ajuda & 4 & 18,2 \\
Com alguma ajuda & 11 & 50 \\
É incapaz & \multicolumn{2}{c}{} \\
\hline Vai a lugares distantes onde exige condução & 2 & 9,1 \\
Sem ajuda & 10 & 45,4 \\
Com alguma ajuda & 10 & 45,4 \\
É incapaz & & \\
\hline Faz compra de alimentos, roupas e outros & 3 & 13,6 \\
Sem ajuda & 5 & 22,7 \\
Com alguma ajuda & 14 & 63,6 \\
É incapaz & & 13,6 \\
\hline Prepara a própria refeição & 3 & 31,8 \\
Sem ajuda & 7 & 54,5 \\
Com alguma ajuda & 12 & 18,2 \\
É incapaz & & 36,4 \\
\hline Faz limpeza e arrumação da casa & 4 & 45,4 \\
Sem ajuda & 8 & 22,7 \\
Com alguma ajuda & 10 & 13,6 \\
É incapaz & \multicolumn{2}{c}{63,6} \\
\hline Lida com as próprias finanças & 5 & \\
Sem ajuda & 3 & \\
Com alguma ajuda & 14 & \\
É incapaz & & \\
\hline
\end{tabular}

A doença de Alzheimer é uma doença neurodegenerativa progressiva, ou seja, gera uma perda gradual da função cognitiva e danifica o desempenho de atividades da vida diária, culminando na perda de autonomia e dependência total de outras pessoas. Com base nisso, o grau de dependência dos idosos foi avaliado pela escala de atividades de vida diária (AVDs) básica e instrumental. A Avaliação Total AVD indica que 4 idosos, 18,2\%, possuem independência para AVDI (20 a 26 pontos), 14 idosos $(63,6 \%)$ tem dependência parcial para AVDI (08 a 19 pontos) e 4 idosos, 18,2\%, com dependência total para AVDI (menos de 7 pontos). Em pesquisa similar, Luzardo, Gorini e Silva (2006), expõem que $55,6 \%$ dos pacientes apresentaram dependência importante, $22,2 \%$ dependência parcial e 22,2\% independência. 
Tabela 6 - Processo de Diagnóstico dos portadores de Alzheimer. Londrina - 2007

\begin{tabular}{lcc}
\hline \multicolumn{1}{c}{ Condições } & $\boldsymbol{n}$ & \% \\
\hline Há quanto tempo iniciaram as alterações de memória? & 11 & 50 \\
24-36 meses & 5 & 22,7 \\
$42-60$ meses & 6 & 27,3 \\
$84-192$ meses & 5 & 22,7 \\
\hline Serviço responsável pelo diagnóstico & 8 & 36,4 \\
Hosp. Particular & 9 & 40,9 \\
Convênio & & \\
Ambulatório de Especialidades & 7 & 31,8 \\
\hline Profissional responsável pelo diagnóstico & 15 & 68,2 \\
Geriatra & & \\
Neurologista & 12 & 54,5 \\
\hline Há quanto tempo foi confirmado o diagnóstico? & 6 & 27,3 \\
12-24 meses & 4 & 18,2 \\
32-36 meses & & \\
$84-120$ meses & & \\
\hline
\end{tabular}

A maioria dos diagnósticos foi confirmada no ambulatório de especialidades por neurologistas, depois de 1 a 2 anos após do início dos esquecimentos, indicando a dificuldade dos profissionais em diagnosticar a doença e a demora dos familiares em procurar um serviço de saúde. Esses problemas ocorrem, pois os pacientes, familiares e profissionais de saúde subestimam os sintomas iniciais, considerando-os como alterações típicas da senilidade (WANNMACHER, 2005).

Quanto às condições de tratamento dos 22 idosos pesquisados, tem-se que 17 (77,3\%) fazem uso de medicação, o qual é fornecido pelo SUS em 100\% dos casos. Todavia, $100 \%$ dos idosos não realizam nenhum tipo de tratamento não farmacológico. As medicações citadas pelos familiares foram Rivastigmina e Donepezil, e apenas um paciente associava os dois medicamentos. Esses fármacos, incluindo a galantamina, pertencem ao grupo dos inibidores das colinesterases de segunda geração e atualmente são os mais utilizados no tratamento da doença de Alzheimer, nos estágios leves a moderado e mostraram-se eficazes na prevenção e no controle de sintomas comportamentais e psicológicos (ENGELHARDT et al., 2005).

O tratamento visa a melhorar as funções cognitivas, comportamentais e psicológicas (WANNMACHER, 2005), por isso é necessário conhecer as alterações e evolução da DA, a fim de escolher a terapêutica e intervenção mais efetiva.

Segundo estudos a DA passa por três estágios de evolução. A fase inicial dura em média de 2 a 3 anos e seu principal sintoma é o comprometimento da memória recente e desorientação de tempo e espaço. A fase intermediária dura em média de 2 a 10 anos e apresenta comprometimento da memória remota, dificuldades em resolver problemas e atividades operativas, afetando as atividades básicas e instrumentais da vida diária. Os pacientes manifestam sintomas comportamentais, alteração na marcha, movimentos lentificados, afasia, agnosia, vocabulário restrito repetido e desorganizado. A fase avançada, com duração aproximada de oito a doze anos, caracteriza-se por total dependência, evidenciada pela perda da capacidade para realizar atividades básicas e instrumentais e imobilidade, 
responsável pelas complicações que acabam levando o paciente a morte (CUNHA, 1999; FRIDMAN et al., 2004).

Sendo assim, uma abordagem terapêutica múltipla, incluindo intervenções farmacológicas e não farmacológicas, é essencial para aumentar a qualidade de vida do paciente e do cuidador. É necessário o acompanhamento multiprofissional e integrado com nutricionistas, fisioterapeutas, fonoaudiólogos, psicólogos, terapeutas ocupacionais, assistente social e enfermeiro (FRIDMAN et al., 2004).

Outras intervenções podem ser instituídas como adequação do ambiente doméstico, tornando-o mais seguro, fácil de ser percorrido e mais agradável ao paciente. Estabelecer rotinas de atividades, incluindo deambulação, exercícios físicos, atividades sociais e intelectuais, também é importante.

A família é peça fundamental para a efetividade do tratamento e do sucesso da recuperação do idoso no domicílio, pois é responsável pelo cuidado (BOTTINO et al., 2002). Esta encontra muitas dificuldades para cuidar do idoso demenciado, justificado pela falta de preparação e treinamento adequados. A equipe de saúde deve orientar o cuidador e familiar sobre o curso e prognóstico da doença e realizar grupos de auto-ajuda, os quais possibilitam a troca de experiências e sentimentos e diminuem a ansiedade. Além disso, é preciso fazer a capacitação de cuidados institucionais e domiciliares, ajudando o familiar a enfrentar problemas cotidianos. A manutenção desses cuidados é mantida através de programas de treinamento, supervisão e assessoria (ARGIMON; TRENTINI, 2006; LUZARDO; GORINI; SILVA, 2006).

Um problema vivenciado pelos cuidadores é a ausência de atendimento especializado e de uma rede de suporte de saúde que atenda aos idosos com demência (LUZARDO; WALDMAN, 2004). O apoio às famílias é oferecido pelos planos de saúde e serviços privados, que beneficiam uma minoria.
Devido ao aumento do número de idosos dependentes em decorrência das doenças crônico-degenerativas, a Política Nacional do Idoso preconizou, pela Lei 8842/1994, que é competência do SUS desenvolver ações para atender as necessidades básicas dos idosos, através da parceria entre familiares, cuidadores profissionais, sociedade, entidades governamentais e não governamentais (BRASIL, 1994).

Todavia essa atitude não é observada na prática e, assim, o Estado deve ampliar o acesso aos serviços de saúde existentes para idosos e instituir programas práticos e efetivos, a fim de atender a população idosa em crescimento e suas famílias (LUZARDO; GORINI; SILVA, 2006).

Uma limitação do presente estudo é o número de idosos entrevistados o que impede a extrapolação dos resultados. Entretanto a realidade aqui apresentada mostra carências existentes na atenção a idosos portadores de DA. Novos estudos precisam ser desenvolvidos para uma avaliação mais ampla dos problemas aqui identificados.

\section{Conclusão}

$\mathrm{O}$ dados apresentados demonstraram que entre os idosos entrevistados, há ainda uma carência de recursos terapêuticos que são indicados na atenção ao idosos portadores de DA. As análises dos dados indicam que apesar dos avanços já realizados nos serviços de atenção a idosos com DA, os idosos entrevistados ainda não possuem acesso a uma assistência interdisciplinar e integral.

A sociedade e as organizações governamentais não podem continuar delegando a família toda responsabilidade pelo cuidado de idosos, especialmente em situações de alta dependência como os portadores de Alzheimer. Deve ser estabelecida uma relação de co-responsabilidade, na qual família e estado ofereçam todos os recursos materiais e humanos necessários para uma atenção integral e humanizada. 


\section{Referências}

ALMEIDA, O. P. Mini exame do estado mental e o diagnóstico de demência no Brasil. Arquivos de NeuroPsiquiatria, São Paulo, v. 56, n. 3-B, p. 605-612, 1998.

ARGIMON, I. L.; TRENTINI, C. M. Apresença da doença de alzheimer e suas repercussões na dinâmica familiar. Revista Brasileira de Ciências do Envelhecimento Humano, Passo Fundo, v. 3, n. 1, p. 98-105, 2006.

BRASIL. LEI n ${ }^{\circ} 8.842$, de 4 de janeiro de 1994. Dispõe sobre a política nacional do idoso, cria o Conselho Nacional do Idoso e dá outras providências. Disponível em $\quad<$ http://www.pge.sp.gov.br/centrodeestudos/ bibliotecavirtual/dh/volume\%20i/idosolei8842.htm>. Acesso em: 29 jun. 2008.

BRASIL. Ministério da Saúde. Secretaria de Atenção à Saúde. Departamento de Atenção Básica. Envelhecimento e saúde da pessoa idosa. Brasília: Ministério da Saúde, 2006. (Série A. Normas e Manuais Técnicos) (Cadernos de Atenção Básica, n. 19). Disponível em: $<$ http://189.28.128.100/portal/saude/cidadao/area. cfm?id_area $=153>$. Acesso em: 29 jun. 2008.

BOtTINO, C. M. C.; CARVALHO, I. A. M.; ALVAREZ, A. M. M. A.; AVILA, R.; ZUKAUSKAS, P. R.; BUSTAMANTE, S. E. Z.; ANDRADE, F. C.; HOTOTIAN, S. R.; SAFFI, F.; CAMARGO, C. H. P. Reabilitação cognitiva em pacientes com doença de alzheimer: relato de trabalho em equipe multidisciplinar. Arquivos de Neuro-Psiquiatria, São Paulo, v. 60, n. 1, p. 70-79, 2002.

CARAMELLI, P.; BARBOSA, M. T. Como diagnosticar as quatro causas mais freqüentes de demência? Revista Brasileira de Psiquiatria, São Paulo, v. 24, Supl. I, p. 7-10, 2002.

CUNHA, R. Musicoterapia na abordagem do idoso. 1999. Monografia (Especialização) - Universidade Tuiuti do Paraná. Disponível em: http://www.fapr.br/site/pesquisa/ revista/artigos/rosemyriamcunha.pdf $>$. Acesso em: 29 jun. 2008.

ENGELHARDT, E.; BRUCKI, S. M. T.; CAVALCANTI, J. L. S.; FORLENZA, O. V.; LAKS, J.; VALE, F. A. C. Tratamento da doença de alzheimer: recomendações e sugestões do departamento científico de neurologia cognitiva e do envelhecimento da academia brasileira de neurologia. Arquivos de Neuro-Psiquiatria, São Paulo, v. 63, n. 4, p. 1104-1112, 2005.
FRIDMAN, C.; GREGÓRIO, S. P.; DIAS NETO, E.; OJOPI, É. P. B. Alterações genéticas na doença de alzheimer. Revista da Associação Médica Brasileira, São Paulo, v. 31, n. 1, p. 19-25, 2004.

GIROLAMI, U.; ANTHONHY, D. C.; FROSCH, M. P. O sistema nervoso central. In: STANLEY, L. R.; KUMAR, V.; COTRAN, R. S. Patologia estrutural e funcional. 6. ed. Rio de Janeiro: Guanabara Koogan, 2000. p. 11871189 .

HERRERA JUNIOR, E.; CARAMELI, P.; NITRINI, R. Estudo epidemiológico populacional de demência na cidade de Catanduva - Estado de São Paulo-Brasil. Revista de Psiquiatria Clínica, São Paulo, v. 25, n. 2, p. 70-73, 1998.

LUZARDO, A. R.; GORINI, M. I. P. C.; SILVA, A. P. S. S. Características de idosos com doença de alzheimer e seus cuidadores: uma série de casos em um serviço de neurogeriatria. Texto e Contexto Enfermagem, Florianópolis, v. 15, n. 4, p. 587-594, 2006.

LUZARDO, A. R.; WALDMAN, B. F. Atenção ao familiar cuidador do idoso com doença de alzheimer. Acta Scientiarum-Ciências da Saúde, Maringá, v. 26, n. 1, p. 135-145, 2004.

MACHADO, J. C. B. Doença de alzheimer. In: FREITAS, E. V.; PY, L.; NERI, A. L.; CANÇADO, F. A. X.; GORZONI, O. M. L.; ROCHA, S. M. Tratado de geriatria e gerontologia. 2. ed. Rio de Janeiro: Guanabara Koogan, 2006. p. 260-279.

NAKATAN, A. Y. K.; SOUTO, C. C. S.; PAULETTE, L. M.; MELO, T. S.; SOUZA, M. M. Perfil dos cuidadores informais de idosos com déficit de autocuidado atendidos pelo programa de saúde da família. Revista Eletrônica de Enfermagem, Goiânia, v. 5, n. 1, 2003. Disponível em: $<$ http:/www.fen.ufg.br/revista $>$. Acesso em: 12 maio 2008.

NITRINI, R.; CARAMELLI, P.; BOTTINO, C. M. C.; DAMASCENO, B. P.; BRUCKI, S. M. D.; ANGHINAH, R. Diagnóstico de doença de alzheimer no Brasil: critérios diagnósticos e exames complementares. Arquivos de Neuro-Psiquiatria, São Paulo, v. 63, n. 3-A, p. 713-719, 2005 .

SANTOS, S. S. C.; PELZER, M. T.; RODRIGUES, M. C. T. Condições de enfrentamento dos familiares cuidadores de idosos portadores de doença de alzheimer. Revista Brasileira de Ciências do Envelhecimento Humano, Passo Fundo, v. 4, n. 2, p. 114-126, 2007. 
VILELA, L. P.; CARAMELLI, P. A doença de alzheimer na visão de familiares de pacientes. Revista da Associação Médica Brasileira, São Paulo, v. 52, n. 3, p. 148-152, 2006.
WANNMACHER, L. Demência: evidências contemporâneas sobre a eficácia dos tratamentos. Organização Pan-Americana da Saúde, Brasília, v. 2, n. 4, 2005. Disponível em: <http://www.opas.org.br/ medicamentos/site/UploadArq/HSE_URM_DEC_0305. pdf $>$. Acesso em: 11 maio 2010.

Recebido em 18 de setembro de 2008 - Received on September 18, 2008. Aceito em 13 de maio de 2009 - Accepted on May 13, 2010. 